ISSN 1409-2441

www.ucr.ac.cr
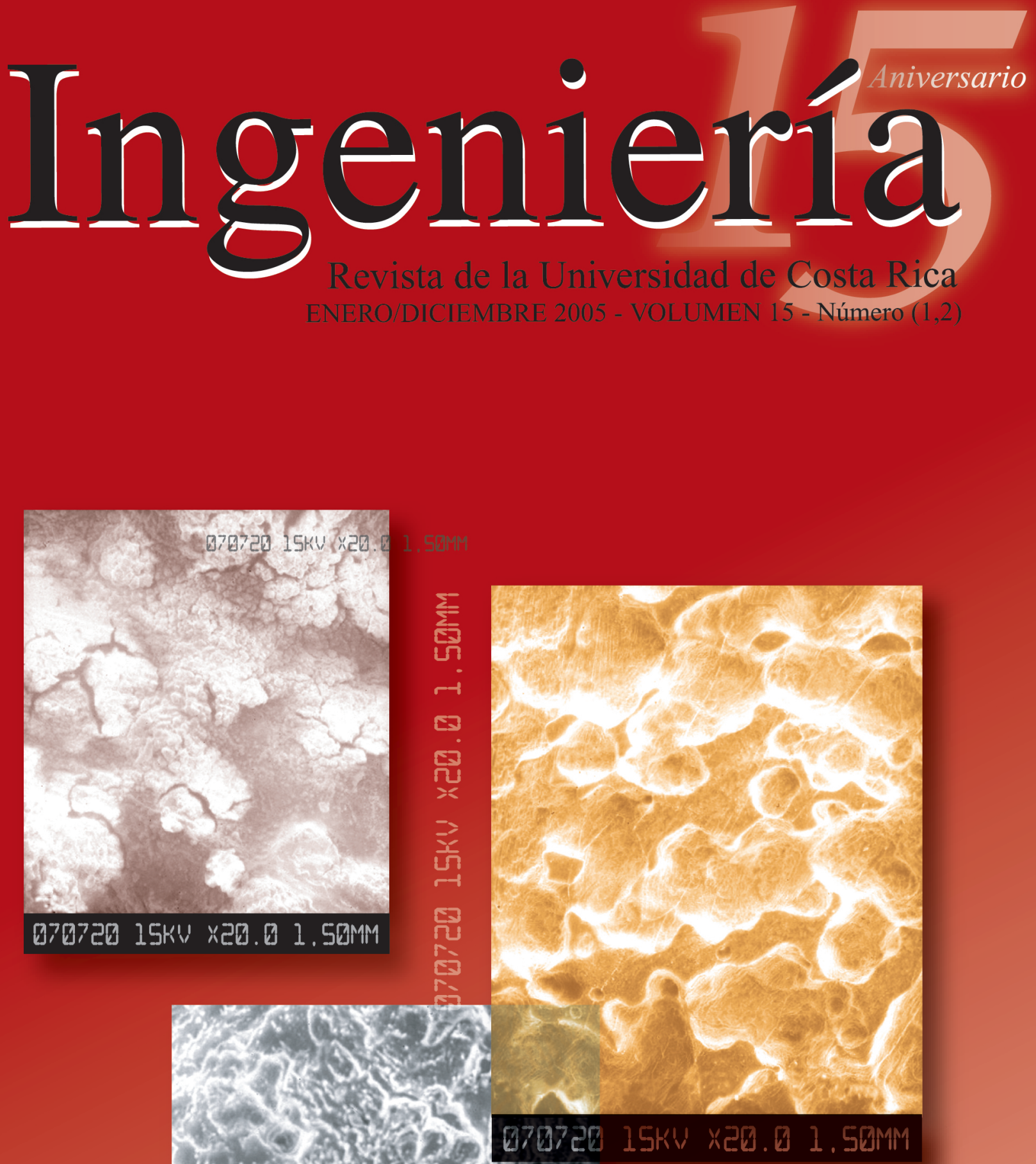

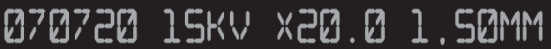

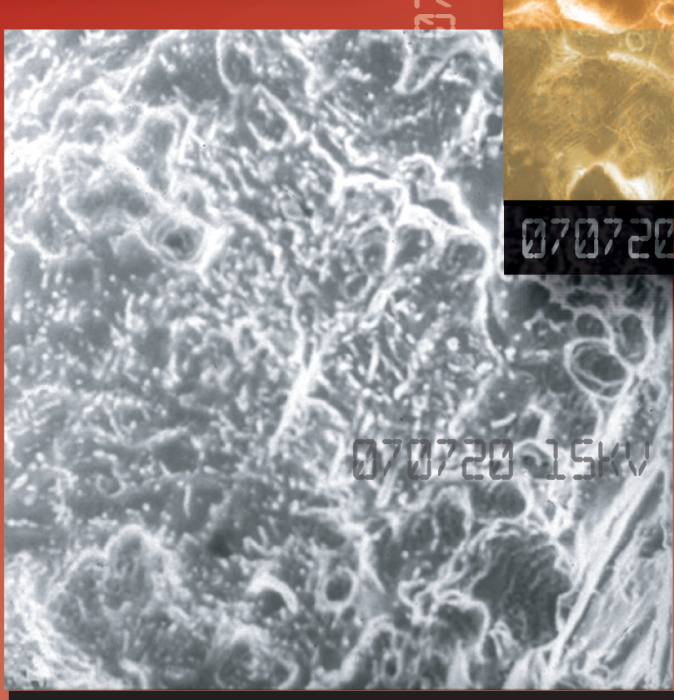

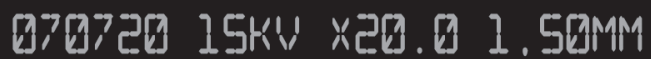




\title{
ESTIMACIÓN DEL DESEMPEÑO IAE ÓPTIMO DE LOS REGULADORES Y SERVOMECANISMOS PID
}

\author{
Víctor M. Alfaro Ruiz
}

\section{Resumen}

Se presenta en forma gráfica la variación del valor de la integral del error absoluto $I A E$, el error máximo $E_{m a x}$, el tiempo de asentamiento al $2 \% T_{a 2}$ y la robustez de los sistemas de control, con controladores PID optimizados con el criterio $I A E$, operando como reguladores y como servomecanismos, en función del tiempo muerto normalizado del modelo.

Se deducen ecuaciones para el cálculo de la $I A E$, el $E_{\max }$ y el $T_{a 2}$ para estos sistemas de control. Mediante ejemplos, se demuestra la utilidad de las ecuaciones obtenidas para la estimación del comportamiento dinámico del sistema de control.

Palabras clave: controladores $P I D$, sintonización, $I A E$

\section{Abstract}

How the integral of the absolute error $I A E$, the maximum error $E_{\max }$, the $2 \%$ settling time $T_{a 2}$ and the robustness of a $I A E$ optimized PID control system, change with the model normalized time delay, are presented in graphical form.

Equations to obtain the $I A E, E_{\max }$ and $T_{a 2}$ are derived for these control systems. The examples presented allow verifying the useful of the equations for the performance estimation of the control system.

Key words: $P I D$ controllers, tuning, IAE

Recibido: 24 de enero del 2005 / Aprobado: 29 de setiembre del 2005

\section{INTRODUCCIÓN}

Existen varias reglas para la sintonización óptima de los reguladores y servomecanismos que utilizan controladores PID (Figura 1). Todas estas se basan en un modelo identificado para el proceso controlado y en la optimización de un criterio de desempeño, normalmente la integral de una función del error. Algunas de las más conocidas son las reglas de López (López, 1967) y las de Rovira (Rovira, 1969). Recientemente, (Alfaro, 2003) determinó nuevas ecuaciones para el cálculo de los parámetros óptimos de los controladores PID operando como reguladores (cambio en la perturbación z) y como

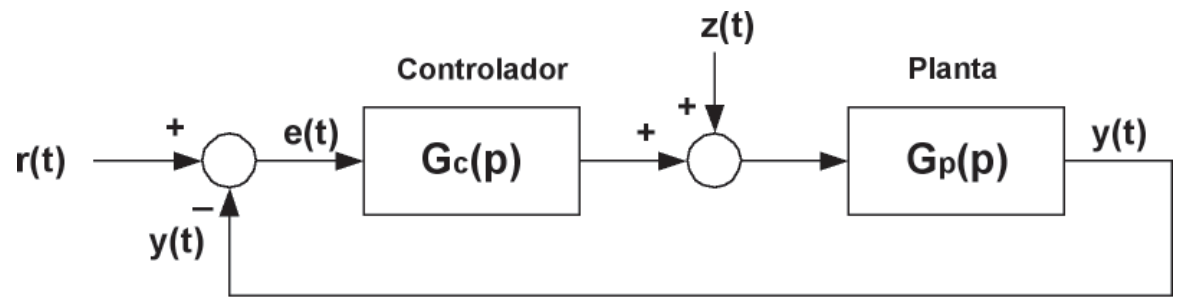

Figura 1. Sistema de control retroalimentado Fuente: El autor 
servomecanismos (cambio en el valor deseado $r$ ) bajo el criterio $I A E$, a partir de un modelo de primer orden más tiempo muerto como planta, que amplían el ámbito de aplicación de este tipo de reglas.

Como es de suponer, con la utilización de estos procedimientos de sintonización, se espera que el comportamiento del sistema sea óptimo bajo el criterio de desempeño utilizado. Sin embargo, es importante también, tener alguna indicación de cuáles podrían ser otras características del comportamiento dinámico del sistema de control.

Se presenta adelante la forma como varía la integral del error absoluto, el error máximo, el tiempo de asentamiento y la robustez del sistema de control, en función del tiempo muerto normalizado de la planta, para reguladores y servomecanismos $P I D$ optimizados con el criterio IAE. A partir de esta información, se derivaron ecuaciones para su estimación.

\section{SINTONIZACIÓN ÓPTIMA}

\subsection{Modelo de la planta}

El método propuesto supone que la dinámica del proceso se puede representar adecuadamente por un modelo de primer orden más tiempo muerto de la forma

$$
G_{p}(s)=\frac{k_{p} e^{-t_{m} s}}{\tau s+1}
$$

\subsection{Criterio de desempeño}

El criterio de desempeño optimizado es la Integral del Error Absoluto definida como

$$
I A E=\int_{0}^{\infty}|e(t)| d t
$$

en donde el error está dado por

$$
e(t)=r(t)-y(t)
$$

\subsection{Parámetros del controlador}

Las ecuaciones para el cálculo de los parámetros óptimos ( $\left.K_{c}, T_{i}, T_{d}\right)$ de un controlador PID-Ideal son (Alfaro, 2003):

- Regulador

$$
\begin{aligned}
& k_{p} K_{c}=0,2068+1,1597 \tau_{o}^{-1,0158} \\
& \frac{T_{i}}{\tau}=-0,2228+1,3009 \tau_{o}^{0,5022} \\
& \frac{T_{d}}{\tau}=0,3953 \tau_{o}{ }^{0,8469}
\end{aligned}
$$

- Servomecanismo

$$
k_{p} K_{c}=0,3295+0,7182 \tau_{o}^{-0,9971}
$$

$$
\frac{T_{i}}{\tau}=0,9781+0,3723 \tau_{o}^{0,8456}
$$

$$
\frac{T_{d}}{\tau}=0,3416 \tau_{o}^{0,9414}
$$

en donde $\tau_{o}=t_{m} / \tau\left(0,05 \leq \tau_{o} \leq 2,0\right)$ es el tiempo muerto normalizado de la planta.

\section{DESEMPEÑO DE LOS LAZOS DE CONTROL}

Como se comentó anteriormente, las reglas de sintonización desarrolladas optimizan el criterio IAE. Como es usual con las reglas de sintonización, estas no dan indicación alguna sobre las características del desempeño dinámico del sistema de control optimizado, por lo que se obtuvieron expresiones que permiten estimar algunas de ellas. 


\subsection{Variación de las características dinámicas}

Mediante una simulación digital utilizando el programa VisSim ${ }^{\mathrm{TM}}$ (Visual Solutions, 1999) se determinó el valor del criterio de desempeño (integral del error absoluto - IAE), así como el error máximo $\left(E_{\max }\right)$ y el tiempo de asentamiento al $2 \%\left(T_{a 2}\right)$, de las respuestas del regulador $(\Delta z=1)$ y del servomecanismo $(\Delta r=1)$ para un tiempo muerto normalizado variando de 0,05 a 2,0 (ámbito de aplicación de las ecuaciones de sintonización determinadas).

El estudio de los resultados permitió determinar que en el caso de los reguladores, el valor de la $I A E z$ y el tiempo de asentamiento $T_{a 2 z}$, son proporcionales a la constante de tiempo del proceso y que el valor del error máximo $E_{\operatorname{maxz}}$ y la $I A E z$ son proporcionales a la ganancia de la planta.

En el caso de los servomecanismos se determinó que, el valor de la $I A E r$ es proporcional a la constante de tiempo de la planta, pero no depende de la ganancia de esta, como tampoco el sobrepaso máximo $E_{\text {maxz }}$. Igual que en el caso de los reguladores, para los servomecanismos el tiempo de asentamiento $T_{a 2 r}$ es proporcional a la constante de tiempo de la planta.

Considerando lo anterior, los resultados obtenidos se normalizaron de la siguiente forma:

$$
\begin{aligned}
& I A E_{z n}=\frac{I A E_{z}}{k_{p} \tau}, I A E_{r n}=\frac{I A E_{r}}{\tau} \\
& E_{\text {max } z n}=\frac{E_{\max z}}{k_{p}}, \quad E_{\max r n}=E_{\max r} \\
& T_{a 2 z n}=\frac{T_{a 2 z}}{\tau}, \quad T_{a 2 r n}=\frac{T_{a 2 r}}{\tau}
\end{aligned}
$$

La constante de tiempo, del modelo ( $\tau$ ), se empleó para normalizar la escala de tiempo y su ganancia $(k p)$ para normalizar la magnitud del error en el caso del regulador solamente.
En la Figura 2, se muestran, identificados con un símbolo (*), los resultados obtenidos para el regulador, en la Figura 3, los correspondientes

al servomecanismo, para los valores de $\tau_{o}$ probados.

Como se puede apreciar en estos gráficos, el tiempo muerto tiene un efecto adverso en todos los casos. Al aumentar el tiempo muerto se deterioran todos lo indicadores del comportamiento dinámico del sistema de control; aumenta la $I A E$, el error máximo (o el sobrepaso máximo en el caso del servomecanismo) y el tiempo de asentamiento.

El valor óptimo de la $I A E$, aumenta casi en forma lineal con el tiempo muerto relativo del proceso y en el caso de los reguladores, su magnitud es directamente proporcional a la ganancia de la planta.

El error máximo $\left(E_{\max }\right)$ para la operación como regulador, es la característica de desempeño que más se ve afectada por el incremento en el tiempo muerto y puede ser apreciable si la ganancia de la planta es alta. En contraste con esto, el sobrepaso máximo de los servomecanismos, varía entre un $5 \%$ y un $15 \%$, valores que pueden considerarse bajos.

Los gráficos anteriores también permiten sacar conclusiones sobre la incidencia de los otros parámetros del modelo, en el desempeño del lazo de control. Es evidente que los sistemas de control evolucionan según la escala de tiempo del proceso controlado. Si se normaliza la escala de tiempo como $t^{\prime}=t / \tau$, las respuestas de todas las plantas con la misma relación $\tau_{O}$ serían idénticas.

Por ejemplo, si se considera el caso de una planta con un tiempo muerto aparente igual a la

mitad de su constante de tiempo ( $\left.\tau_{o}=0,5\right)$, el tiempo de asentamiento, al $2 \%$ del sistema de control, operando como regulador sería.

$$
t_{a 2} \approx 3,5 \tau \text {. }
$$


Entonces, el sistema de control de una planta con esta característica y una constante de tiempo de dos minutos, tardaría siete minutos en regresar la variable controlada a su valor deseado ante un cambio en una perturbación, y el de una planta con una constante de tiempo de dos segundos, tardaría siete segundos.

\subsection{Ecuaciones para la estimación del desempeño}

Para poder estimar el valor de los índices de desempeño de los lazos de control cuyo controlador se haya optimizado empleando el criterio IAE, se realizó un ajuste de curvas mediante mínimos cuadrados en Matlab® (Matworks, 2002), y se determinaron las siguientes ecuaciones:

- Regulador

$\frac{I A E}{k_{p} \tau \Delta z}=-0,0595+0,9989 \tau_{o}^{1,2507}$

$$
\begin{aligned}
& \frac{E_{\max }}{k_{p} \Delta z}=0,0376+0,8057 \tau_{o}-0,2016 \tau_{o}^{2} \\
& \frac{T_{a}}{\tau}=3,7985 \tau_{o}^{1,0332}, \tau_{o} \leq 0,2 \\
& \frac{T_{a}}{\tau}=-0,7296+7,3912 \tau_{o}^{0,8075}, \tau_{o}>0,2
\end{aligned}
$$

- Servomecanismo

$$
\frac{I A E}{\tau \Delta r}=1,3724 \tau_{o}^{0,9577}
$$$$
\frac{E_{\max }}{\Delta r}=0,0311+0,0288 \tau_{o}+0,0207 \tau_{o}^{2}
$$$$
\frac{T_{a}}{\tau}=3,8518 \tau_{o}^{0,920,} \tau_{o} \leq 1,05
$$

. 

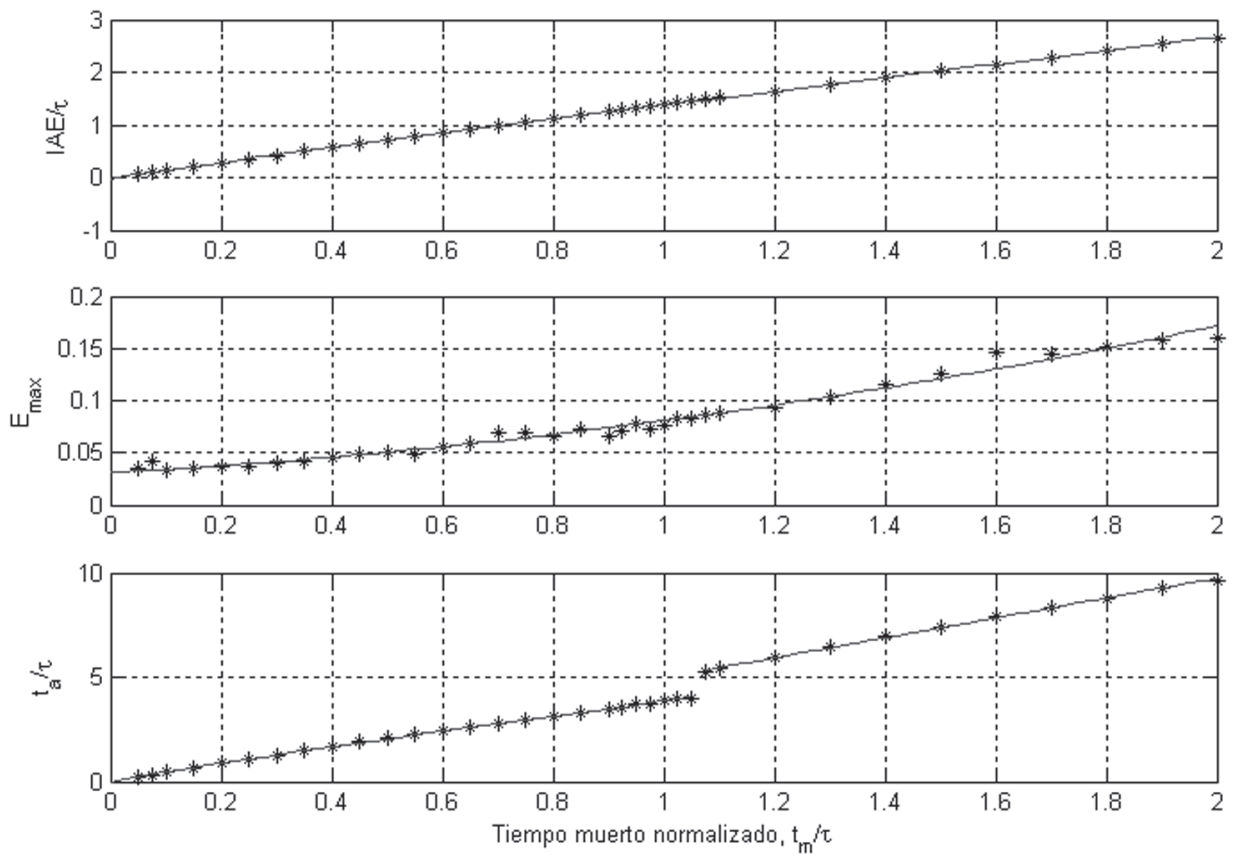

Figura 3. Desempeño del servomecanismo óptimo

Fuente: El autor

$$
\frac{T_{a}}{\tau}=4,9626 \tau_{o}^{0,9725}, \tau_{o}>1,05
$$

En las Figuras 2 y 3, se muestran mediante líneas continuas, los valores predichos por estas ecuaciones.

\section{3 Índices de robustez}

Otro aspecto que es importante considerar al momento de sintonizar un controlador utilizando una regla de sintonización, es la robustez del sistema de control resultante. Esto es, su habilidad para mantener una operación estable ante cambios en las características dinámicas del proceso controlado.

Se definieron los índices de robustez como:

- Índice de robustez en la ganancia
$I R_{k_{p}}=\frac{k_{p u}}{k_{p a}}-1, \quad t_{m}=t_{m a}$

- Índice de robustez en el tiempo muerto

$I R_{t_{m}}=\frac{t_{m u}}{t_{m a}}-1, k_{p}=k_{p a}$

en donde $\left(k_{p a}, t_{m a}\right)$ son los valores de la ganancia y el tiempo muerto del modelo (los utilizados para sintonizar el controlador) y

$\left(k_{p u}, t_{m u}\right)$ los valores de la ganancia y el tiempo muerto que, en forma independiente, hacen oscilatorio el sistema de control. En la Figura 4, se muestra como varían los índices de robustez al variar el tiempo muerto aparente.

Usualmente se considera que un sistema de control es robusto, si tiene índices de robustez 


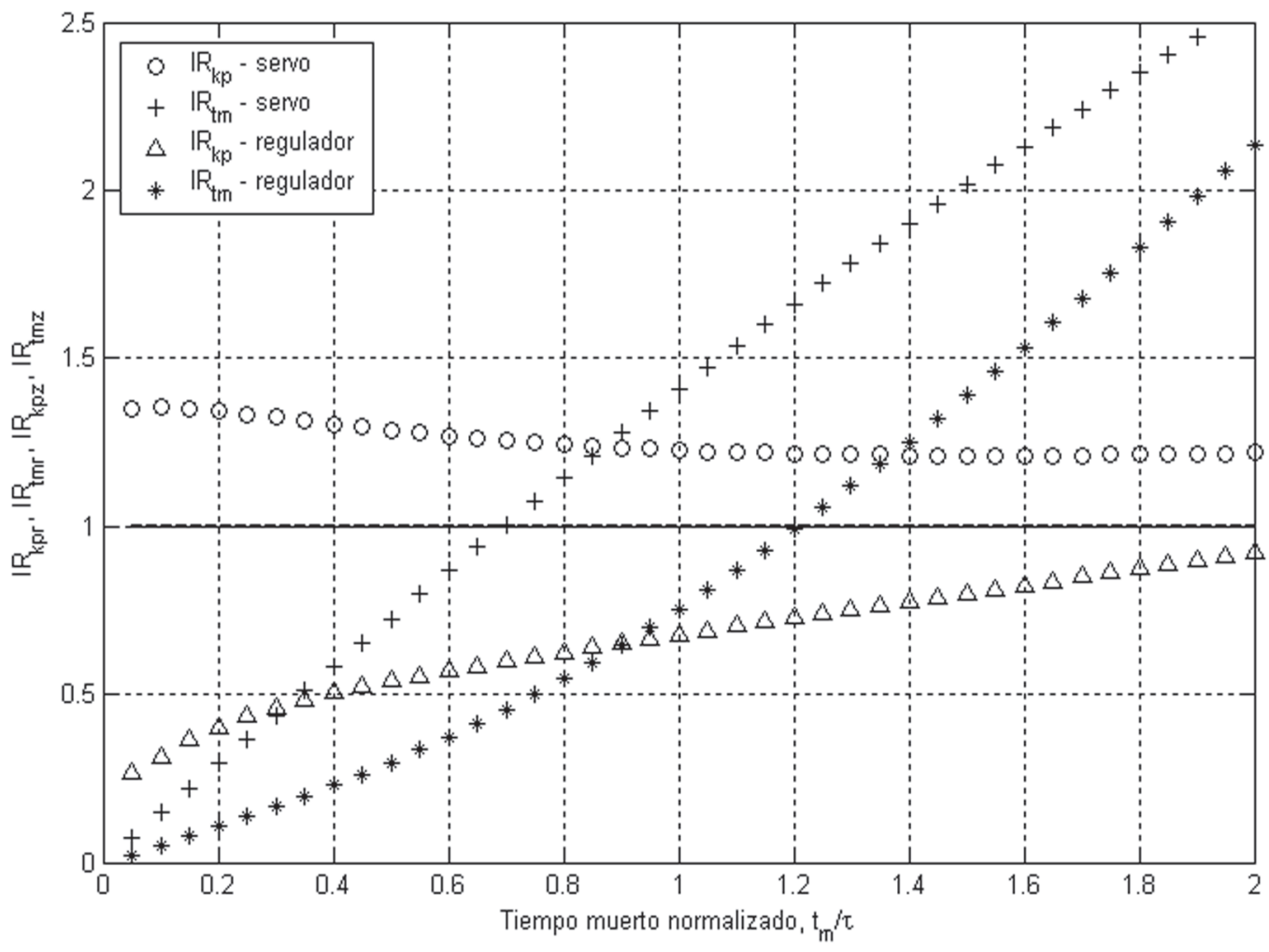

Figura 4. Índice de robustez Fuente: El autor

superiores a uno. Como se puede apreciar, los reguladores optimizados con el criterio IAE no son robustos y los servomecanismos lo son solo para $\tau$ o $>0,70$. Al contrario de lo que sucede con las características dinámicas, en general, al aumentar el tiempo muerto aparente, la robustez del sistema mejora, con la excepción del índice de robustez en la ganancia del servomecanismo, el cual, se mantiene aproximadamente constante.

Lo anterior debe tomarse muy en cuenta, especialmente para los sistemas dominados por

la constante de tiempo $\left(t_{m}<\tau\right)$, ya que en estos casos, el índice de robustez en la ganancia del regulador, por ejemplo, es inferior a 0,6. Esto indica que incrementos pequeños en la ganancia de la planta, harían muy oscilatoria la respuesta del sistema.

\section{EJEMPLOS}

\subsection{Planta de cuarto orden}

Para verificar la utilidad de las ecuaciones determinadas, para la estimación de las características de desempeño de los sistemas de control sintonizados con el criterio IAE, se empleó como planta de prueba (Aström \& Hägglund, 2000)

$G_{p 1}(s)=\frac{2}{(s+1)(0,5 s+1)(0,25 s+1)(0,125 s+1)}$

Para esta, se obtuvo un modelo de primer orden

más tiempo muerto $G_{m 1}(s)$, identificado a partir de la curva de respuesta de la planta mediante el 
método "1/4 - 3/4" (Anexo A) y otro identificado a partir de la información última $\left(\mathrm{K}_{\mathrm{cu}}=3,3750\right.$,

$\left.\mathrm{T}_{\mathrm{u}}=2,2214\right), G_{m 2}(s)$ (Anexo B). Los parámetros de estos modelos se muestran en el Cuadro 1.

Cuadro 1. Parámetros de los modelos

$\begin{array}{ccc} & G_{m 1} & G_{m 2} \\ k_{p} & 2,0 & 2,0 \\ \tau & 1,247 & 2,360 \\ t_{m} & 0,691 & 0,608 \\ & \text { Fuente: El autor } & \end{array}$

Utilizando los dos modelos identificados, se determinaron los parámetros del controlador $P I D$ para un funcionamiento como regulador, ecuaciones (4) a (6), y para un funcionamiento como servomecanismo, ecuaciones, (7) a (9), los cuales se muestran en los Cuadros 2 y 3.

Cuadro 2. Parámetros del controlador (regulador)

$\begin{array}{ccc} & G_{c 1} & G_{c 2} \\ K_{c} & 1,16 & 2,40 \\ T_{i} & 0,93 & 1,03 \\ T_{d} & 0,30 & 0,30\end{array}$

Cuadro 3. Parámetros del controlador (servomecanismo)

$\begin{array}{ccc} & G_{c 1} & G_{c 2} \\ K_{c} & 0,81 & 1,55 \\ T_{i} & 1,50 & 2,59 \\ T_{d} & 0,24 & 0,22 \\ & \text { Fuente: El autor } & \end{array}$

Además, utilizando las ecuaciones (13) a (16) para el regulador, y las (17) a (20) para el servomecanismo, se estimaron las características del desempeño de los lazos de control, las cuales se muestran en los Cuadros 4 y 5 .

Cuadro 4. Desempeño estimado del regulador

$\begin{array}{ccc} & G_{m 1} & G_{m 2} \\ I A E & 1,042 & 0,584 \\ E_{\text {max }} & 0,844 & 0,464 \\ T_{a 2} & 4,812 & 4,113 \\ & \text { Fuente: } \text { El autor } & \end{array}$

Cuadro 5. Desempeño estimado del servo

$\begin{array}{ccc} & G_{m 1} & G_{m 2} \\ I A E & 0,972 & 0,884 \\ E_{\max } & 0,053 & 0,040 \\ T_{a 2} & 2,790 & 2,610\end{array}$

Fuente: El autor

En las cuadros anteriores se puede apreciar que tanto los parámetros del controlador como la estimación de los índices de desempeño se ven afectados por el modelo utilizado. Para verificar los datos obtenidos, se determinaron las características dinámicas de los sistemas de control, con los dos controladores anteriores, empleando tanto la planta original como los modelos, las cuales se muestran en los Cuadros 6 y 7.

Cuadro 6. Desempeño del regulador

\begin{tabular}{ccccc} 
& $G_{c 1} G_{p}$ & $G_{c 2} G_{p}$ & $G_{c 1} G_{m 1}$ & $G_{c 2} G_{m 2}$ \\
$I A E$ & 1,112 & 0,490 & 0,986 & 0,534 \\
$E_{\text {max }}$ & 0,594 & 0,390 & 0,871 & 0,475 \\
$T_{a 2}$ & 6,292 & 3,778 & 4,942 & 4,397 \\
& \multicolumn{5}{c}{ Fuente: El autor }
\end{tabular}

Cuadro 7. Desempeño del servomecanismo

\begin{tabular}{ccccc} 
& $G_{c 1} G_{p}$ & $G_{c 2} G_{p}$ & $G_{c 1} G_{m 1}$ & $G_{c 2} G_{m 2}$ \\
$I A E$ & 1,035 & 0,994 & 0,967 & 0,860 \\
$E_{\text {max }}$ & 0,052 & 0,138 & 0,053 & 0,037 \\
$T_{a 2}$ & 3,233 & 6,197 & 2,791 & 2,612 \\
& \multicolumn{5}{c}{ Fuente: El autor }
\end{tabular}

El Cuadro 6, muestra claramente, que en el caso del regulador, se logró un mejor desempeño, menor $I A E$, con la planta de prueba, con el controlador sintonizado empleando el modelo identificado partir de la información última ( $\left.G_{m 2}\right)$.

Comparando los resultados de los Cuadros 6 y 4, se puede apreciar, que la estimación hecha del desempeño del regulador, con base en el modelo $G_{m 2}$ es bastante acertada y en todo caso ligeramente conservadora.

En el Cuadro 7, se puede notar también que se logra un menor índice IAE, si se sintoniza el controlador con los parámetros obtenidos 
utilizando el modelo $G_{m 2}$, sin embargo en este caso, la diferencia entre el desempeño de los dos controladores no es significativa. Se nota en cambio una diferencia apreciable en las otras características dinámicas del lazo de control.

Si se comparan los resultados del Cuadro 7 con los del Cuadro 5, se puede apreciar que las características dinámicas estimadas para el servomecanismo, con los dos modelos, permiten tener una buena indicación de las características dinámicas de la respuesta, con excepción del tiempo de asentamiento con el controlador $G_{c 2}$.

Como se puede apreciar en las Figuras 5 y 6, las respuestas del sistema de control obtenidas empleando los modelos, difieren de las obtenidas con los mismos controladores y la planta de prueba. Esta diferencia es mayor cuando se emplea el modelo identificado a partir de la curva de reacción. Esto indica, que aunque los modelos puedan representar fielmente la respuesta de lazo abierto, en un caso y las condiciones de oscilación en el otro, no necesariamente, reflejan el comportamiento dinámico del proceso bajo condiciones de control en lazo cerrado.

\subsection{Planta de polos múltiples}

Para verificar la incidencia que pudiera tener la planta sobre la estimación de las características dinámicas del sistema de control, se realizaron pruebas similares con un sistema de polo múltiple de orden ocho dado por

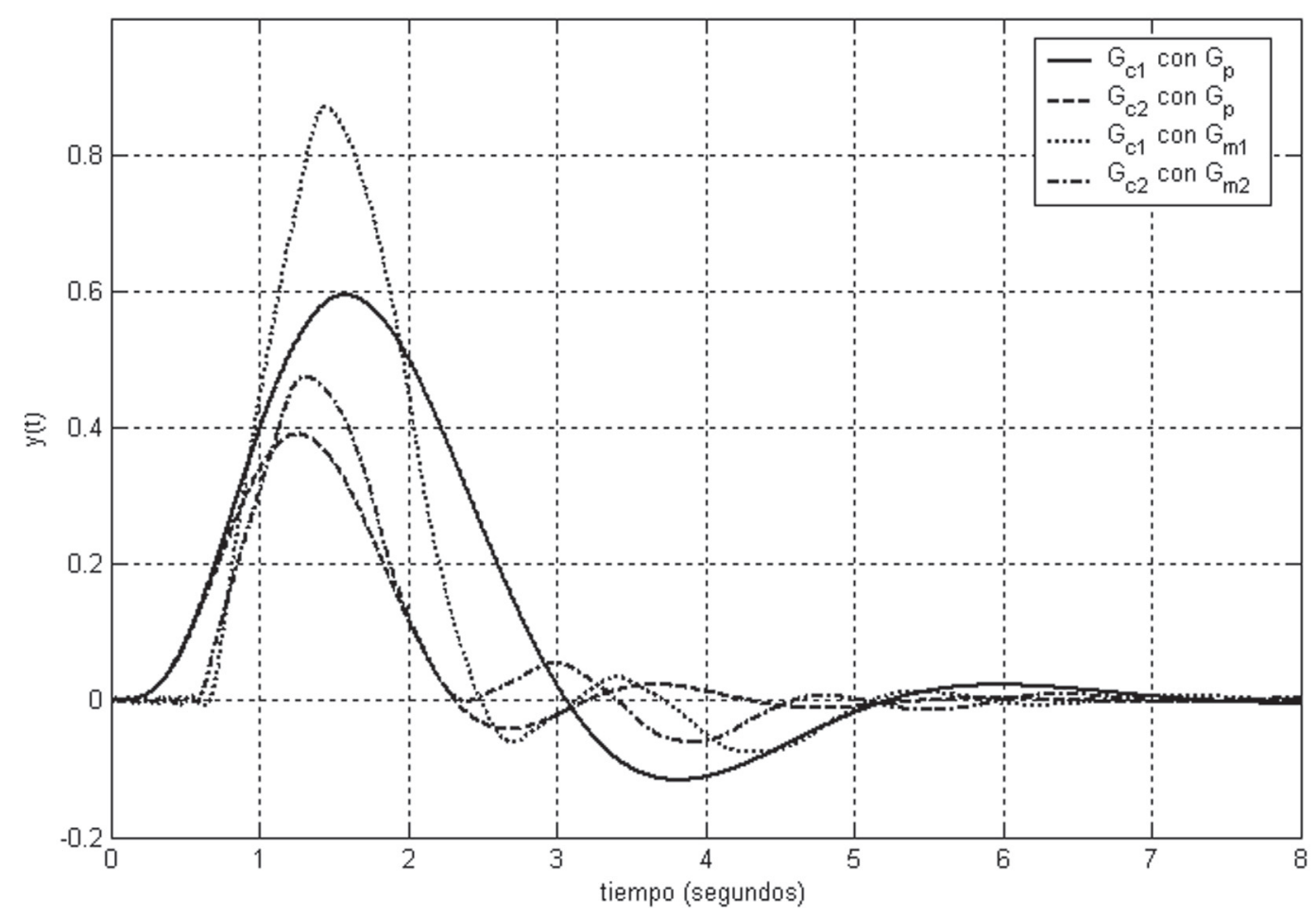

Figura 5. Respuesta del regulador (Cambio en $z(t)$ )

Fuente: El autor 


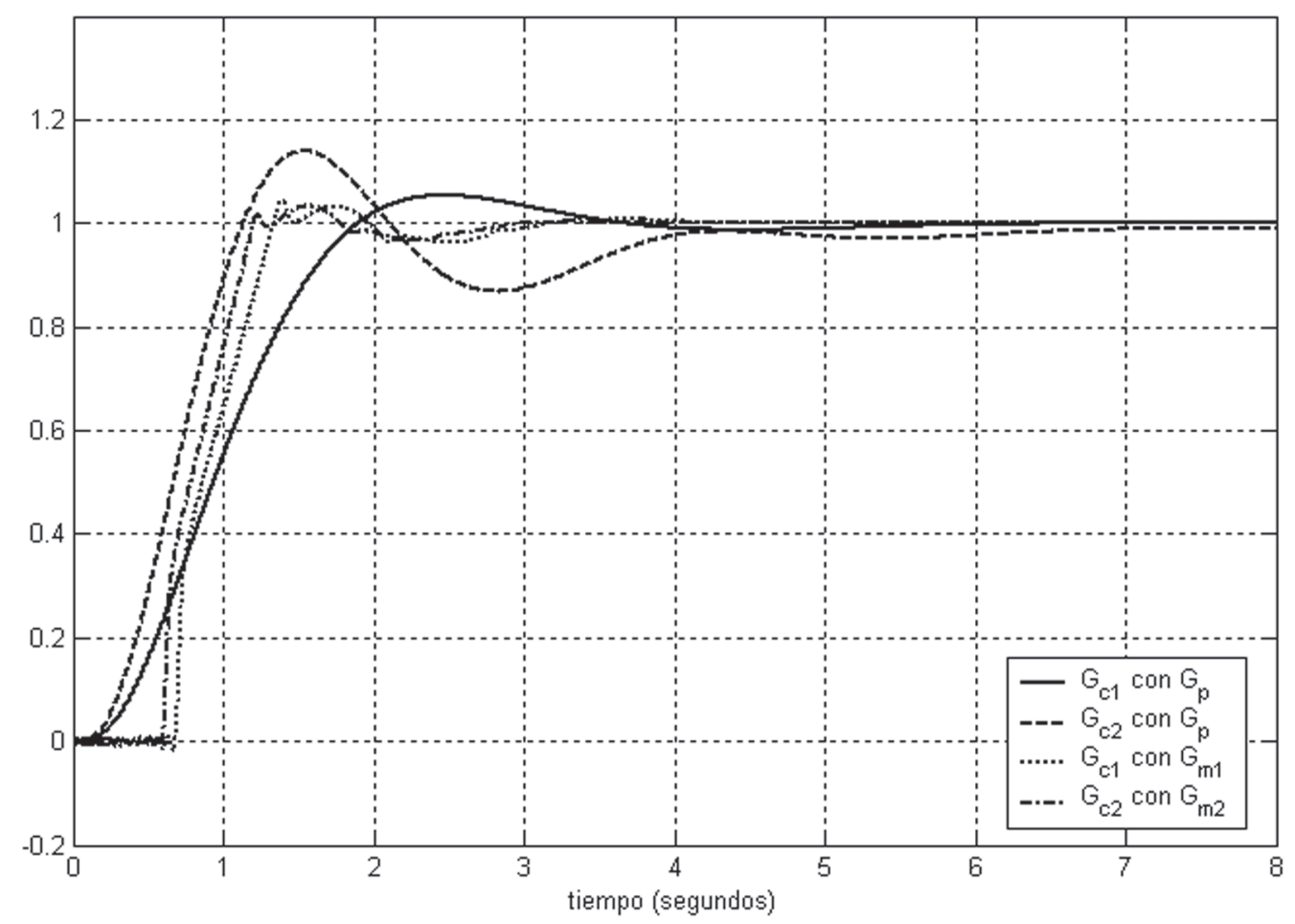

Figura 6. Respuesta del servomecanismo (Cambio en $r(t)$ )

Fuente: El autor

$$
G_{p 2}(s)=\frac{1}{(s+1)^{8}}
$$

Los modelos, los parámetros de los controladores y los valores de las características dinámicas de las respuestas como regulador y como servomecanismo se muestran en los Cuadros 8 al 14.

Cuadro 8. Parámetros de los modelos

$\begin{array}{ccc} & G_{m 1} & G_{m 2} \\ k_{p} & 1,0 & 1,0 \\ \tau & 3,39 & 3,85 \\ t_{m} & 4,98 & 5,14 \\ & \text { Fuente: El autor } & \end{array}$

Cuadro 9. Parámetros del controlador (regulador)

$\begin{array}{ccc} & G_{c 1} & G_{c 2} \\ K_{c} & 0,99 & 1,07 \\ T_{i} & 4,59 & 4,93 \\ T_{d} & 1,86 & 1,94 \\ & \text { Fuente: El autor } & \end{array}$

Cuadro 10. Parámetros del controlador (servo)

$\begin{array}{ccc} & G_{c 1} & G_{c 2} \\ K_{c} & 0,82 & 0,87 \\ T_{i} & 5,06 & 5,60 \\ T_{d} & 1,16 & 1,73\end{array}$


Cuadro 11. Desempeño estimado del regulador

$\begin{array}{ccc} & G_{m 1} & G_{m 2} \\ I A E & 5,28 & 5,29 \\ E_{\text {max }} & 0,79 & 0,75 \\ T_{a 2} & 31,71 & 33,13 \\ & \text { Fuente: El autor } & \end{array}$

Cuadro 12. Desempeño estimado del servo

$\begin{array}{ccc} & G_{m 1} & G_{m 2} \\ I A E & 6,72 & 6,97 \\ E_{\max } & 0,12 & 0,10 \\ T_{a 2} & 24,45 & 25,31 \\ & \text { Fuente: El autor } & \end{array}$

Cuadro 13. Desempeño del regulador

\begin{tabular}{ccccc} 
& $G_{c 1} G_{p}$ & $G_{c 2} G_{p}$ & $G_{c 1} G_{m 1}$ & $G_{c 2} G_{m 2}$ \\
$I A E$ & 6,74 & 6,48 & 5,34 & 5,36 \\
$E_{\max }$ & 0,72 & 0,69 & 0,77 & 0,74 \\
$T_{a 2}$ & 33,62 & 29,91 & 31,82 & 33,28 \\
& \multicolumn{5}{c}{ Fuente: El autor }
\end{tabular}

Cuadro 14. Desempeño del servomecanismo

\begin{tabular}{ccccc} 
& $G_{c 1} G_{p}$ & $G_{c 2} G_{p}$ & $G_{c 1} G_{m 1}$ & $G_{c 2} G_{m 2}$ \\
$I A E$ & 7,70 & 6,84 & 7,20 & 6,99 \\
$E_{\max }$ & 0,14 & 0,05 & 0,11 & 0,11 \\
$T_{a 2}$ & 25,97 & 21,84 & 16,54 & 25,37 \\
& \multicolumn{5}{c}{ Fuente: El autor }
\end{tabular}

Como se aprecia en los resultados anteriores (Cuadro 11 vs. 13 y Cuadro 12 vs. 14), las ecuaciones deducidas permiten estimar adecuadamente el comportamiento de los sistemas de control, cuando se emplean como planta los modelos utilizados para la sintonización de los controladores.

\section{CONCLUSIONES}

En adición al criterio de desempeño integral seleccionado, al emplear una regla de sintonización para el cálculo de los parámetros de los controladores PID, es conveniente poder estimar algunas de las características que podría tener el sistema de control una vez sintonizado el controlador.
Las ecuaciones (13) a (16) para los reguladores y la (17) a (20) para los servomecanismos, permiten obtener una estimación adecuada del índice de desempeño $I A E$, del error máximo $E_{\text {max }} \mathrm{y}$ del tiempo de asentamiento al $2 \% T_{a 2}$.

Como las ecuaciones derivadas están basadas en los resultados determinados, utilizando un modelo de primer orden más tiempo muerto, las características del desempeño obtenidas con estas, deben de tomarse solo como una aproximación de lo que se podría esperar obtener al poner el controlador a operar con el proceso real. La diferencia entre lo estimado y los resultados reales obtenidos, dependerá de la fidelidad con que el modelo utilizado represente las características dinámicas del proceso controlado.

Además de lo anterior, el estudio permite también concluir, que los resultados obtenidos se ven afectados por el modelo utilizado para el cálculo de los parámetros del controlador. Para el caso particular de las plantas utilizadas en los ejemplos, los mejores resultados se obtuvieron con los modelos identificados a partir de la información crítica del sistema.

\section{SIMBOLOGÍA}

$\tau \quad$ constante de tiempo

$\tau_{\mathrm{o}} \quad$ tiempo muerto relativo

$e(t) \quad$ señal de error

$E_{\max } \quad$ error máximo

$G_{c}(s)$ función de transferencia del controlador

$G_{p}(s)$ función de transferencia del modelo

$K_{c} \quad$ ganancia del controlador

$K_{c u} \quad$ ganancia última

$k_{p} \quad$ ganancia estática

$I A E$ integral del error absoluto

$r(t) \quad$ valor deseado

$s \quad$ variable compleja

$T_{a 2} \quad$ tiempo de asentamiento

$T_{d} \quad$ tiempo derivativo

$T_{i} \quad$ tiempo integral

$t_{m} \quad$ tiempo muerto aparente

$T_{u} \quad$ periodo de oscilación

$y(t) \quad$ variable controlada

$z(t) \quad$ perturbación 


\section{REFERENCIAS BIBLIOGRÁFICAS}

Alfaro, V. M. (2004). IE-432 Laboratorio de Control Automático - Notas de clase. Manuscrito no publicado, Escuela de Ingeniería Eléctrica, Universidad de Costa Rica.

Alfaro, V. M. (2003). Optimización del desempeño de los reguladores y servomecanismos PID. Revista Ingeniería, 13, (1,2): 53-62.

Aström, K. \& Hägglund, T. (2000, abril). Benchmark systems for PID Control. IFAC Workshop on Digital control: Past, Present and Future of PID Control, Terrasa, España.

López, A., Miller, J., Smith, C. \& Murrill, P. (1967). Tuning controllers with error-integral criteria. Instrumentation Technology, November.

Rovira, A., Murrill, P. \& Smith, C. (1969). Tuning controllers for setpoint changes,
Instrumentation \& Control Systems, December, 67-69.

The Matworks, Inc. (2002). MATLAB 6.5 [Programa computacional]. EUA.

Visual Solutions. (1999). VisSim User's Guide Version 3. [Programa computacional]. EUA: Westford, Ma.

\section{SOBRE EL AUTOR}

\section{Víctor M. Alfaro Ruiz}

Profesor Asociado, Departamento de Automática, Escuela de Ingeniería Eléctrica, Universidad de Costa Rica

Apartado postal 2-10, 2060, San José, Costa Rica Teléfono 207.4472

Facsímil 207.4139

Correo electrónico: valfaro@eie.ucr.ac.cr

El presente trabajo fue realizado como parte del proyecto $\mathrm{N}^{\mathrm{o}} 322-\mathrm{A} 3-007$ inscrito en la Vicerrectoría de Investigación de la Universidad de Costa Rica. 


\section{ANEXO A}

Método de identificación “1-4-3/4” (Alfaro, 2004)

Este es un método del tipo de dos puntos. A partir de la curva de reacción del proceso se deben determinar los tiempos requeridos para que la respuesta alcance el $25 \%$ (t25) y el $75 \%$ (t75) del cambio total.

Los parámetros del modelo de primer orden más tiempo muerto se obtienen como:

$k_{p}=\frac{\Delta y}{\Delta u}$

$\tau=0,910\left(t_{75}-t_{25}\right)$

$t_{m}=1,262 t_{25}-0,262 t_{75}$

\section{ANEXO B}

Modelo de POMTM a partir de la información última

Utilizando un procedimiento de oscilación mantenida con un controlador $\mathrm{P}$ o mediante la prueba del relé, se puede determinar la ganancia crítica $K c u$ y el periodo de oscilación Tu del sistema de control. A partir de estos valores se puede identificar un modelo de primer orden más tiempo muerto utilizando las siguientes ecuaciones:

$\tau=\frac{T_{u}}{2 \pi}\left[\left(K_{c u} k_{p}\right)^{2}-1\right]^{1 / 2}$

$t_{m}=\frac{T_{u}}{2 \pi}\left[\pi-\tan ^{-1}\left(\frac{2 \pi \tau}{T_{u}}\right)\right]$

La ganancia se debe de identificar a partir de una prueba de control P como

$k_{p}=\frac{\Delta y}{K_{C}(\Delta r-\Delta y)}$ 